Here, the second term vanishes, and so does the first factor of the first term. Hence $\varphi_{N-1}^{(N-1)}$ is arbitrary. We may then solve for the other components of $\varphi^{(N-1)}$ in terms of $\varphi_{N-1}^{(N-1)}$. Similarly, we may calculate all the vectors $\varphi^{(k)}$ in terms of arbitrary scale factors, which may be chosen so as to normalize the (transformed) eigenvectors $\varphi^{(k)}$. Finally, from $\psi^{(k)}=S \varphi^{(k)}$, we obtain all the eigenvectors of the original problem.

When we do have degeneracy, let us, for definiteness, place one of the repeated roots in the last position of the diagonal. Now we proceed as above, except that for some value $M$ (say) of the index $k$ we will come across a diagonal coefficient in our triangular system of linear equations which vanishes just as the last diagonal element did. Two possibilities are now open. Either the rest of the linear combination (exclusive of the diagonal term) which constitutes the $M$ th equation vanishes, or it does not. If the former is true, then we are entitled to choose for $\varphi_{M}{ }^{(N)}$ another arbitrary number and hence obtain another eigenvector. If the latter is true, then we are forced to make it equal to zero by setting $\varphi_{N}{ }^{(N)}=0$. This causes us to lose one of the basis vectors of the degenerate subspace defined by the repeated root. We may then start as before by setting $\varphi_{M}^{(N)}$ equal to some arbitrary number and solve for the rest of the components of $\varphi^{N}$ as before. The case when we lose one of the eigenvectors belonging to an eigenvalue corresponds to the case in the Jordan canonical form when a 1 appears attached to two equal roots. When there is no 1 attached to a repeated root, we then have the full complement of eigenvectors for that root. Similar considerations apply to roots of higher multiplicity than 2 .

International Business Machines Corporation

J. GReEnStadt

New York, New York

${ }^{1}$ See, for example, R. T. Gregory, $M T A C$, v. 7, 1953, p. 215.

2 F. Murnaghan, Theory of Group Representations. Johns Hopkins Univ. Press. Chap. 1.

3 Unless a suggestion of J. von NEUMANN (private communication) is followed, according to which one attempts to minimize the S.S.A.V. of the sub-diagonal elements in the $m$ th row and $k$ th column between the pivotal subdiagonal element and the diagonal (including the pivotal element itself). This method, however, is much more time-consuming than that proposed in this paper.

\title{
Double Interpolation Formulae and Partial Deriv- atives in Terms of Finite Differences
}

1. Abstract. For making interpolations at different parts of a table, double interpolation formulae for mixed forward, backward, and central differences have been derived. However, these formulae are rather cumbersome and time consuming in use. For interpolation with more than one variable, formulae in terms of the tabular entries $f_{i j}$ directly instead of differences are much simpler to use. SALZER $^{1}$ has derived such a formula for double-forward interpolation in terms of $f_{i j}$. This formula works satisfactorily for interpolation near the head of a table. For the purpose of interpolating at other parts of a table, double interpolation formulae for other than double-forward interpolation formula have been derived.

Expressions for partial derivatives of different orders in terms of both double 
differences and $f_{i j}$ directly, including those needed in the numerical solution of partial differential equations, are also worked out.

2. Double Interpolation Formulae. For making a double interpolation, the tabular values are usually given in a square or rectangular region. Let us divide this region into nine smaller regions as indicated in Fig. 1 by Roman numerals. In order to interpolate values in all nine regions, nine different interpolation

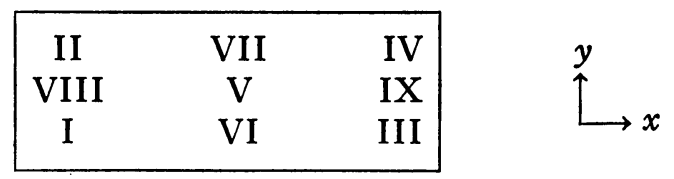

FIG. 1. A tabular values block

formulae are needed. These nine formulae can be further classified into five types, i.e., double-forward, semi-forward, double-backward, semi-central, and doublecentral interpolation formulae, as shown in Table 1. The double interpolation formulae now available are mostly given as functions of double differences. The interpolation formula in terms of double forward differences is known as Biermann's formula or the double Gregory-Newton formula. There are three double

\section{TABLE I. Different types of Interpolation Formulae}

\begin{tabular}{|c|c|c|}
\hline Region & \multicolumn{2}{|c|}{ Interpolation Formula Type } \\
\hline I & Forward $x$, Forward $y$ & Double Forward \\
\hline II & Forward $x$, Backward $y$ & Semi-Forward \\
\hline IV & $\begin{array}{l}\text { Backward } x, \text { Forward } y \\
\text { Backward } x, \text { Backward } y\end{array}$ & Double Backward \\
\hline $\begin{array}{l}\mathrm{V} \\
\mathrm{VI}\end{array}$ & $\begin{array}{l}\text { Central Differences } \\
\text { Central } x \text {, Forward } y\end{array}$ & Double Central \\
\hline $\begin{array}{l}\text { VII } \\
\text { VIII }\end{array}$ & $\begin{array}{l}\text { Central } x \text {, Backward } y \\
\text { Forward } x \text { Central } y\end{array}$ & Semi-Central \\
\hline IX & $\begin{array}{l}\text { Forward } x \text {, Central } y \\
\text { Backward } x \text {, Central } y\end{array}$ & \\
\hline
\end{tabular}

central difference formulae: Stirling-Stirling, Bessel-Bessel and Stirling-Bessel. The other types of the double interpolation formulae as functions of mixed double differences may also be derived easily.

However, these formulae in terms of double differences are rather cumbersome and time consuming in use. For interpolation with more than one variable, formulae in terms of the tabular entries $f_{i j}$ directly instead of double differences should be much simpler to use. Salzer ${ }^{1,2}$ has derived such a formula for double forward interpolation in terms of $f_{i j}$ directly as given in the following equation

$$
f(u, v)=\sum_{i+j=0}^{n}\left(\begin{array}{l}
n-u-v \\
n-i-j
\end{array}\right)\left(\begin{array}{l}
u \\
i
\end{array}\right)\left(\begin{array}{l}
v \\
j
\end{array}\right) f_{i j}
$$

where $u=\frac{x-x_{0}}{h}, v=\frac{y-y_{0}}{k}$ and $h$ and $k$ are the tabular intervals.

$$
\left(\begin{array}{l}
u \\
i
\end{array}\right) \text { denotes } \frac{u(u-1) \cdots(u-i+1)}{i !} \text { with }\left(\begin{array}{l}
u \\
0
\end{array}\right)=1 \text {. }
$$


This formula works satisfactorily for interpolation near the head of a table or in region 1. For the purpose of interpolation in other regions, similar double interpolation formulae of $f_{i j}$ for other than double forward interpolation formula are required.

It is found that by correctly changing the signs of $u$ and $v$ as well as the corresponding subscripts of $f_{i j}$ in Salzer's formula, the interpolation formulae which apply in regions II, III, and IV can be obtained. For example, in order to get a semi-forward interpolation formula which applies in region II, i.e., forward $x$ and backward $y$, one simply substitutes $v$ into $-v$ and $f_{i j}$ into $f_{i,-j}$ in equation (1), and obtains

$$
f(u,-v)=\sum_{i+j=0}^{n}\left(\begin{array}{l}
n-u+v \\
n-i-j
\end{array}\right)\left(\begin{array}{l}
u \\
i
\end{array}\right)\left(\begin{array}{c}
-v \\
j
\end{array}\right) f_{i,-j} .
$$

Similarly, the other semi-forward interpolation formula which applies in region III can be obtained by substituting $-u$ by $u$, and $f_{i j}$ by $f_{-i, j}$ in equation (1). If one changes both $u$ to $-u$ and $v$ to $-v$ and at the same time $f_{i j}$ to $f_{-i,-j}$, a doublebackward interpolation formula results.

These semi-forward and double backward interpolation formulae of $f_{i j}$ thus obtained may be proved to give the same results as those obtained from the corresponding interpolation formulae in terms of double differences.

Any semi-central interpolation formula, for example central $x$ and forward $y$, should make the full use of the tabular entries of both $f_{i j}$ and $f_{-i, j}$. One of the simplest ways of doing this is the following:

$$
f( \pm u, v)=\frac{1}{2}[f(u, v)+f(-u, v)] .
$$

This semi-central interpolation formula can be proved to be correct by the same procedure as employed by Salzer ${ }^{1,2}$ in proving the double forward interpolation formula, provided one can change $f(u, v)$ into $f(u,-v)$ whenever $v<0$, and $f(u, v)$ into $f(-u, v)$ whenever $u<0$. The other three semi-central interpolation formulae can be obtained similarly. Finally, the double-central interpolation formula may be written as follows:

$$
f( \pm u, \pm v)=\frac{1}{4}[f(u, v)+f(u,-v)+f(-u, v)+f(-u,-v)]
$$

Even though the interpolations made by using these semi-central and doublecentral interpolation formulae are very close to those calculated from the double interpolation formulae involving Stirling and Bessel central differences, the results are not exactly the same. Since the interpolation formulae of $f_{i j}$ use the tabular entries more symmetrically than those formulae of central differences do, it may be expected that the interpolation formulae of $f_{i j}$ give more convergent results.

3. Double Differentiation Formulae. In solving a differential equation numerically, either by an iterative method or by the step-ahead method, all the derivative terms of the differential equation should be expressed in terms of finite differences. Formulae for expressing the derivatives of different orders for one independent variable in terms of finite differences have been completely derived for forward, backward, and central differences. ${ }^{3}$ There is thus no difficulty in replacing an ordinary differential equation by a difference equation. 
However, the differential equations involved in solving most physical problems contain two or more independent variables. For solving partial differential equations with two independent variables numerically, formulae for expressing partial derivatives by double differences are required. In order to apply to all parts of a field of interest, the partial derivatives should be expressed by different combinations of double differences. These expressions have been derived and the results are given in Tables II, III, and IV.

In order to avoid the computations of double differences, it again may be worth while to work out some expressions of the partial derivatives in terms of tabular entries $f_{i j}$ directly instead of double differences. These expressions can be obtained from the corresponding double interpolation formulae of $f_{i j}$ previously derived. This is done by differentiating the interpolation formulae of $f_{i j}$ with respect to $u$ and $v$ the required number of times according to the kind and order of the partial derivative of interest and then letting $u=0$ and $v=0$. A general expression for the partial derivative of $f(u, v)$ of order $p+q$ is obtained as follows, where zeros are to be factored out in accordance with usual continuity convention:

$$
\begin{aligned}
& {\left[\frac{\partial^{p}}{\partial u^{p}} \frac{\partial^{q}}{\partial v^{q}} f(u, v)\right]_{u=0, v=0}=\sum_{i+j=0}^{n} \sum_{a+b=p}\left\{\frac{p !}{a ! b !}\left[\left(\begin{array}{l}
0 \\
i
\end{array}\right) \sum_{i_{\alpha}=0}^{i-1} \prod_{\alpha=1}^{a} \frac{1}{-i_{\alpha}}\right] \sum_{l+m=q} \frac{q !}{l ! m !}\right.} \\
& \left.\quad \times\left[(-1)^{l+b}\left(\begin{array}{c}
n \\
n-i-j
\end{array}\right)^{n-i-j-1} \sum_{i_{\alpha}=0}^{\prime} \prod_{\alpha=1}^{l+b} \frac{1}{n-i_{\alpha}}\right]\left[\left(\begin{array}{c}
0 \\
j
\end{array}\right) \sum_{i_{\alpha}=0}^{j-1} \prod_{\alpha=1}^{m} \frac{1}{-i_{\alpha}}\right]\right\} f_{i j}
\end{aligned}
$$

where $p$ and $q$ can be any integer. "'" on the summation sign indicates that no two $i_{\alpha}$ terms can be identical. For instance, when $i=3$ and $a=2$

$$
\sum_{i_{\alpha}=0}^{2} \prod_{\alpha=1}^{2} \frac{1}{-i_{\alpha}}=\frac{1}{0} \frac{1}{-1}+\frac{1}{0} \frac{1}{-2}+\frac{1}{-1} \frac{1}{0}+\frac{1}{-1} \frac{1}{-2}+\frac{1}{-2} \frac{1}{0}+\frac{1}{-2} \frac{1}{-1} .
$$

The general expression of the derivative of $f(u,-v)$ is similar to equation (2) except that $(-1)^{l}$ is removed and a $(-1)^{m}$ term appears in front of $\left(\begin{array}{l}0 \\ j\end{array}\right)$ and also $f_{i j}$ changes to $f_{i,-j}$. Similarly, the expression for the partial derivative of $f(-u, v)$ can be obtained from equation (2) by removing $(-1)^{b}$ and placing a $(-1)^{a}$ term in front of $\left(\begin{array}{l}0 \\ i\end{array}\right)$, and at the same time changing $f_{i j}$ to $f_{-i, j}$. If both of these processes are carried out, an expression of the partial derivative of $f(-u,-v)$ is obtained. The partial derivative of $f( \pm u, v)$ then takes the form

$$
\left[\frac{\partial^{p}}{\partial u^{p}} \frac{\partial^{q}}{\partial v^{q}} f( \pm u, v)\right]_{00}=\frac{1}{2}\left[\left(\frac{\partial^{p}}{\partial u^{p}} \frac{\partial^{q}}{\partial v^{q}} f(u, v)\right)_{00}+\left(\frac{\partial^{p}}{\partial u^{p}} \frac{\partial^{q}}{\partial v^{q}} f(-u, v)\right)_{00}\right]
$$

and

$$
\begin{aligned}
{\left[\frac{\partial^{p}}{\partial u^{p}} \frac{\partial^{q}}{\partial v^{q}} f( \pm u, \pm v)\right]_{00}=\frac{1}{4} } & {\left[\left(\frac{\partial^{p}}{\partial u^{p}} \frac{\partial^{q}}{\partial v^{q}} f(u, v)\right)_{00}+\left(\frac{\partial^{p}}{\partial u^{p}} \frac{\partial^{q}}{\partial v^{q}} f(u,-v)\right)_{00}\right.} \\
& \left.+\left(\frac{\partial^{p}}{\partial u^{p}} \frac{\partial^{q}}{\partial v^{q}} f(-u, v)\right)_{00}+\left(\frac{\partial^{p}}{\partial u^{p}} \frac{\partial^{q}}{\partial v^{q}} f(-u,-v)\right)_{00}\right] .
\end{aligned}
$$




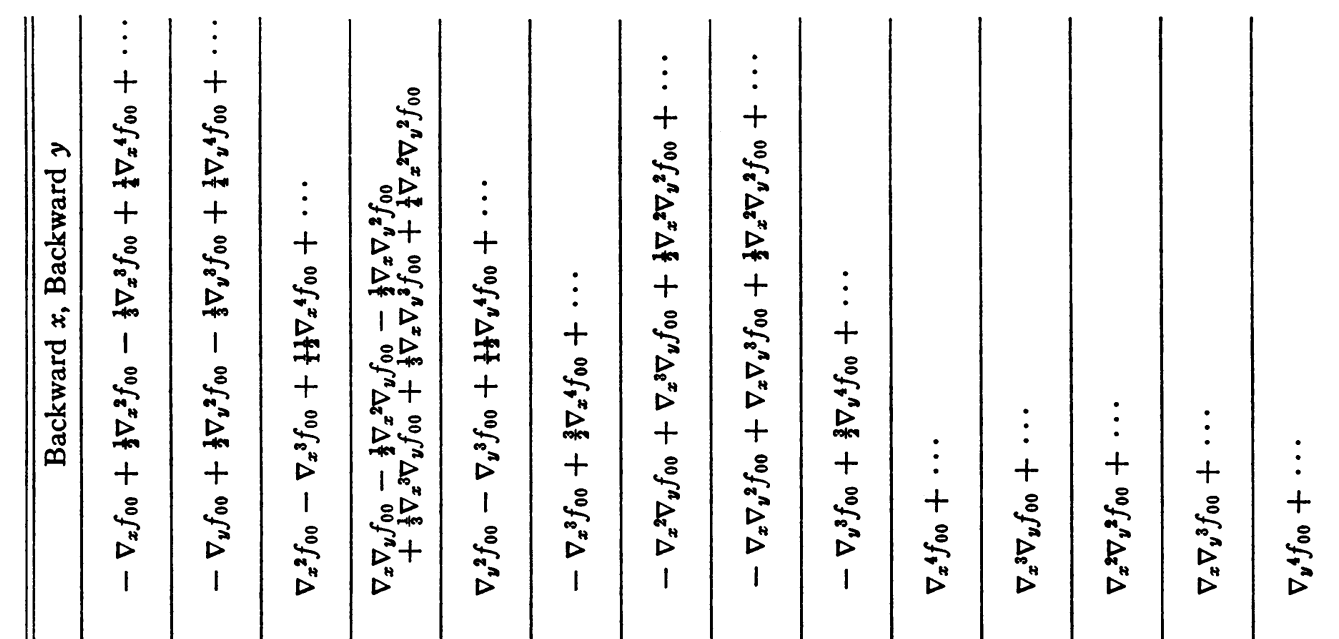

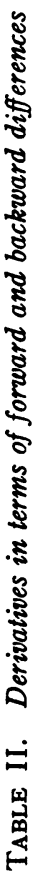

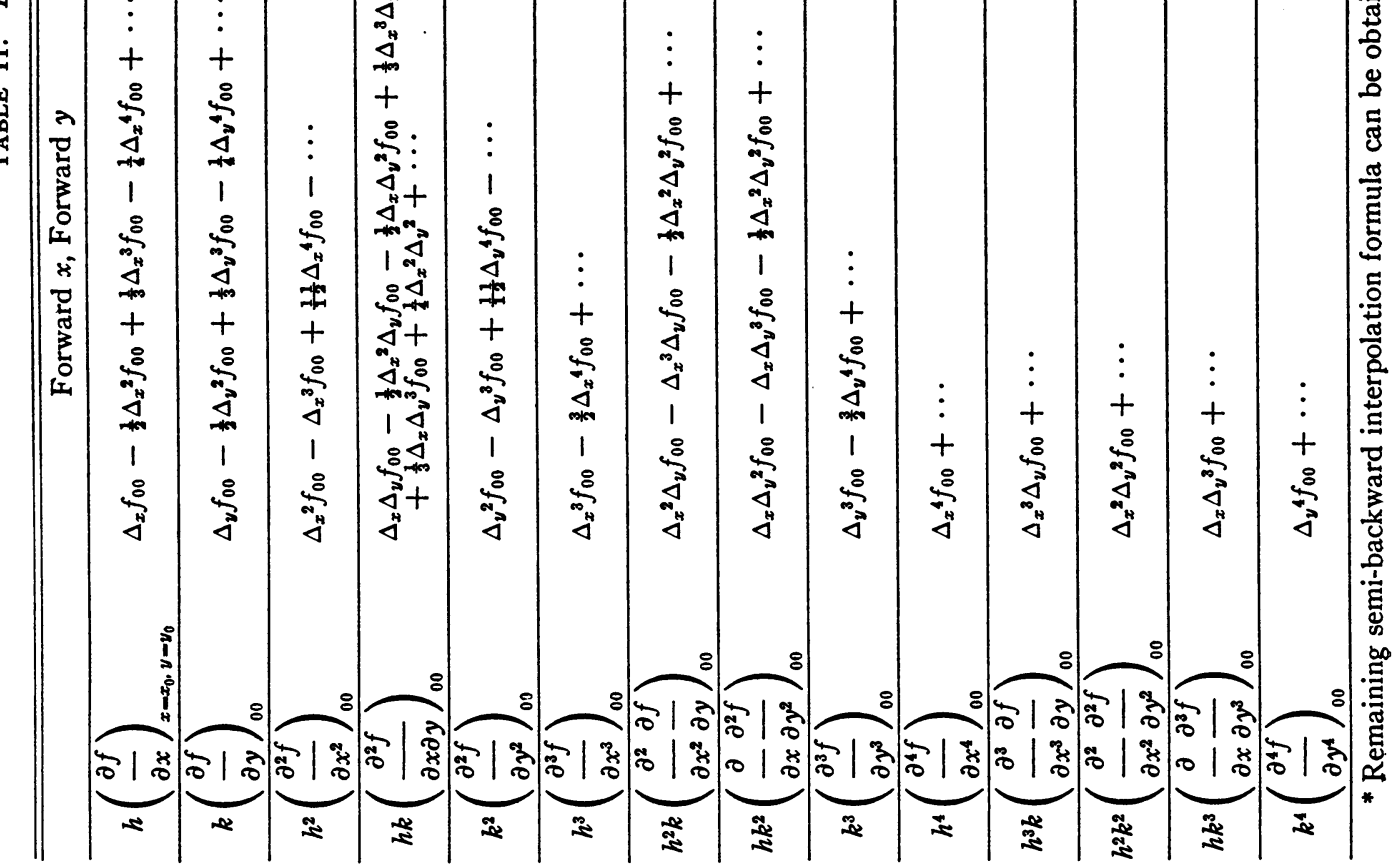




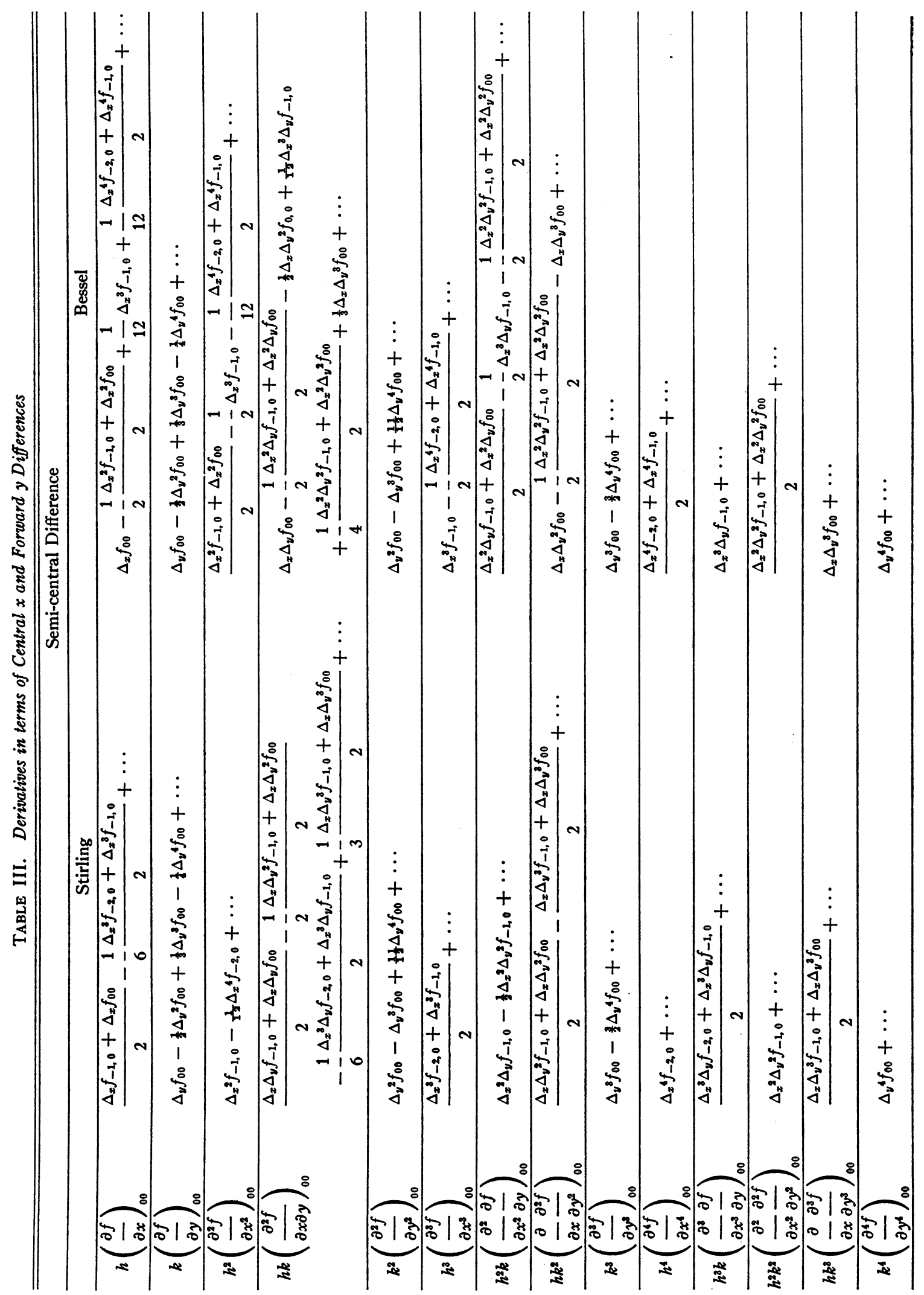




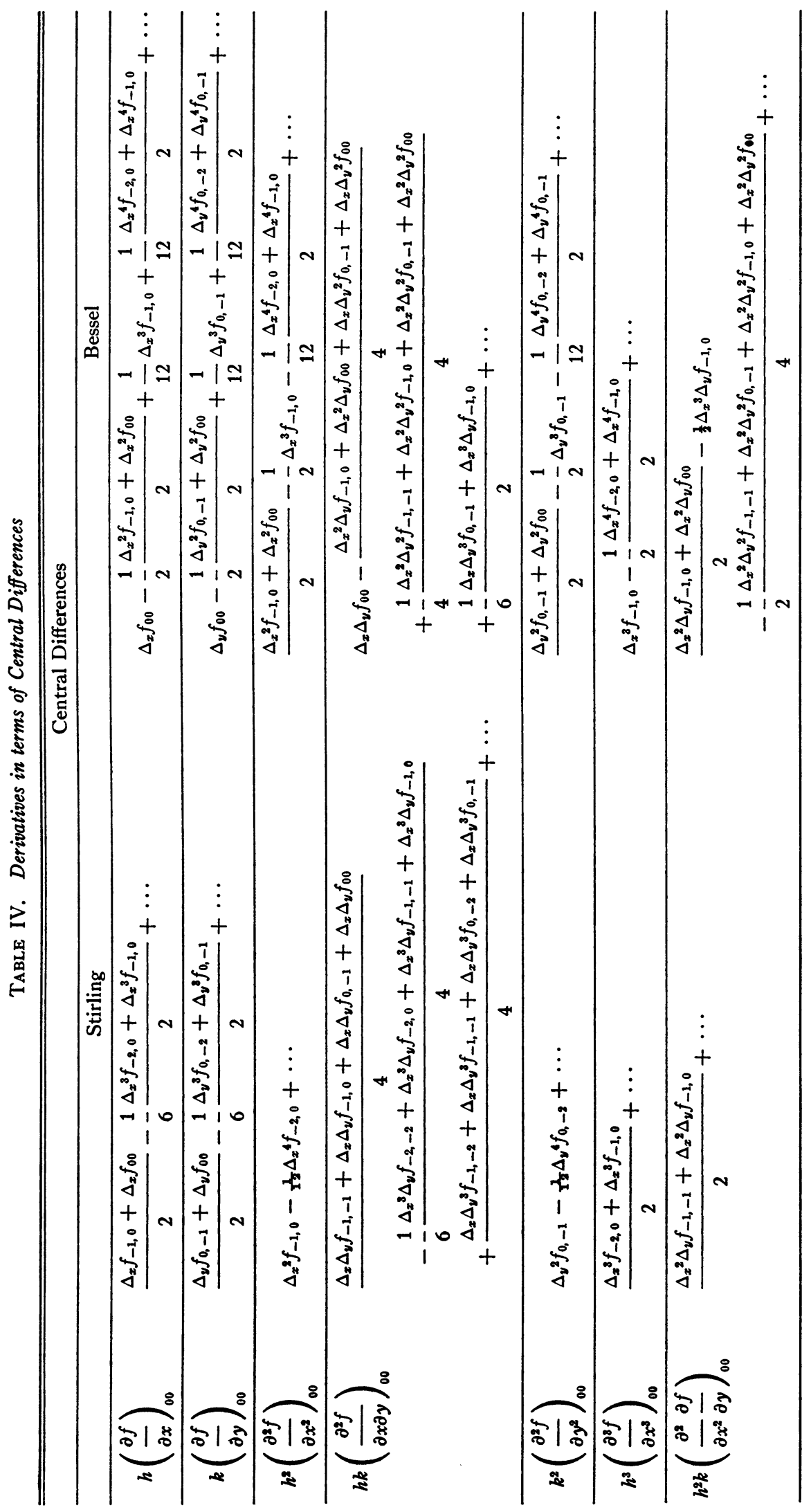




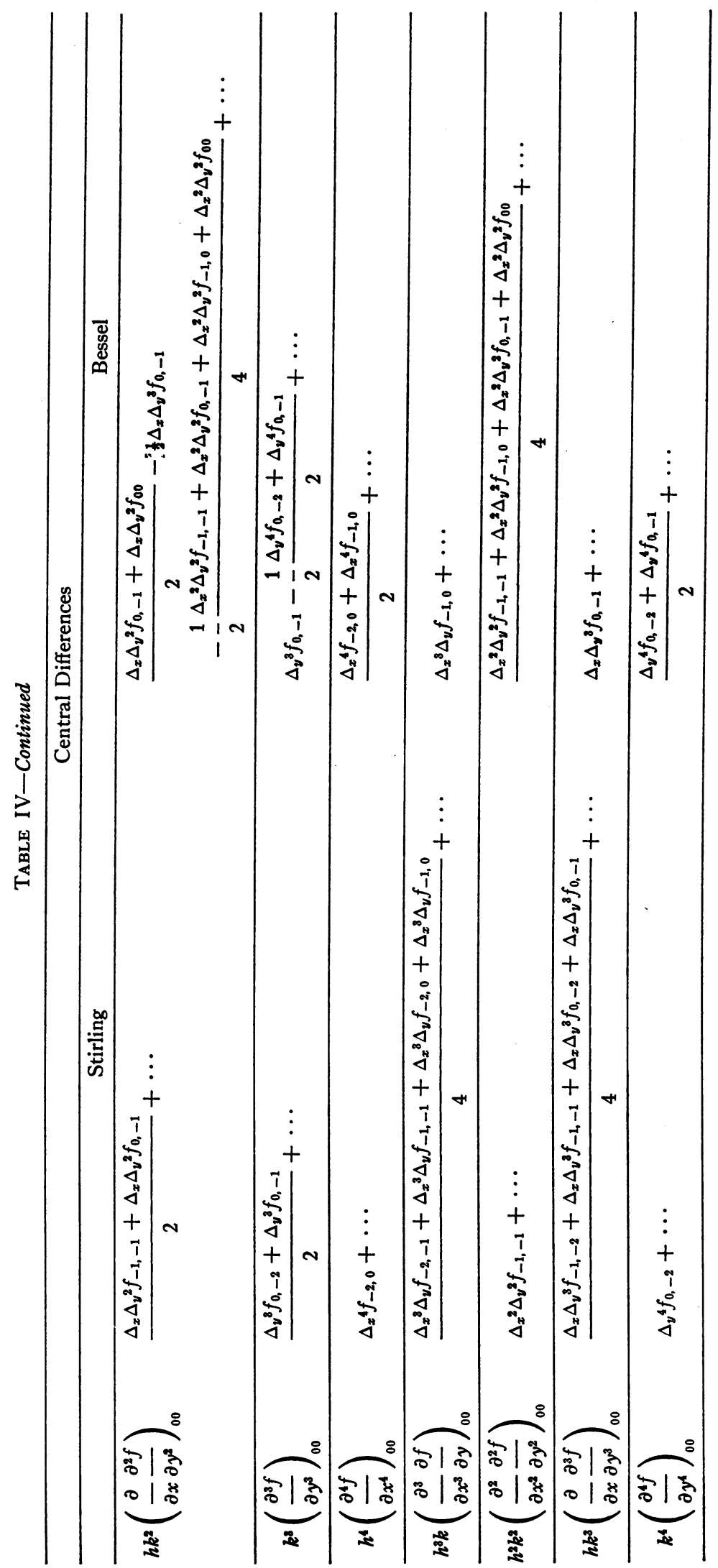




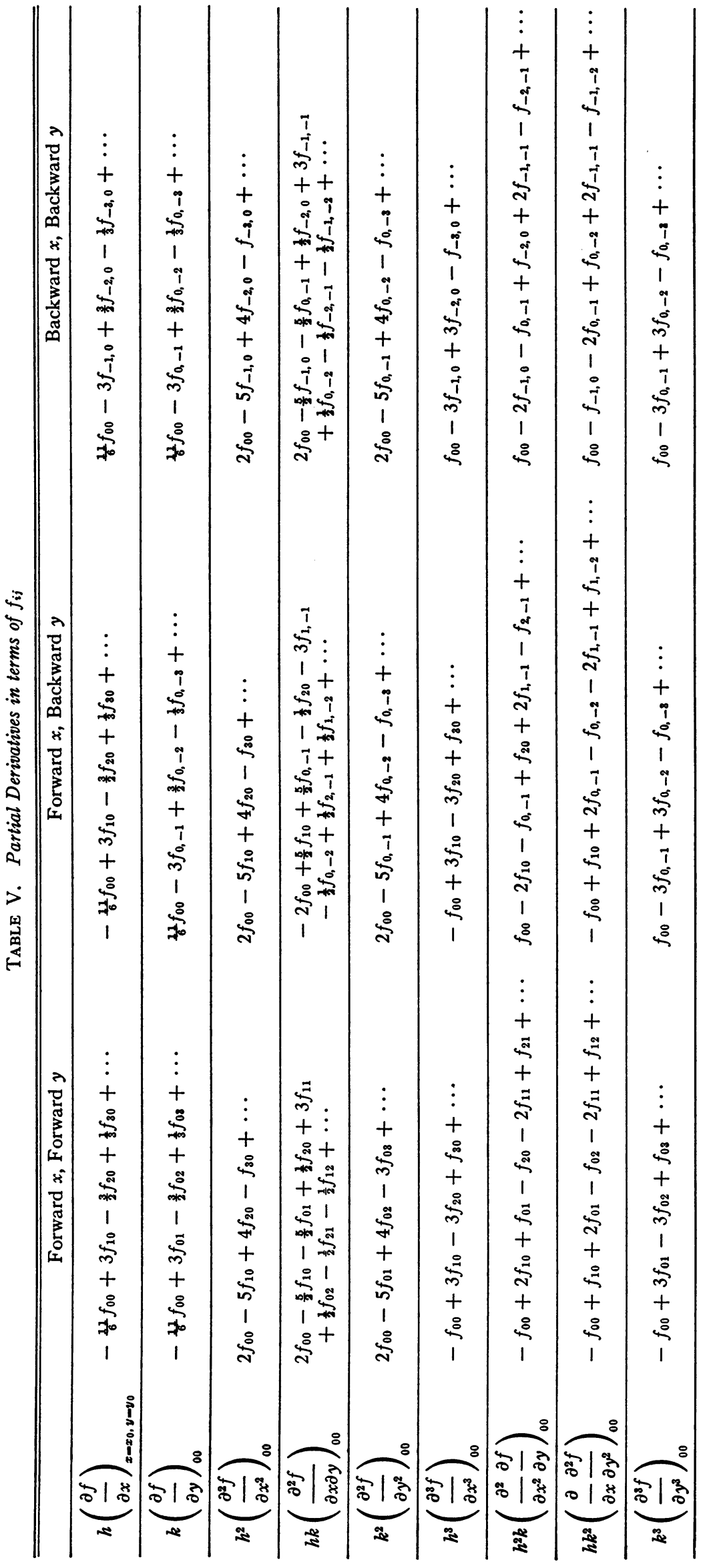




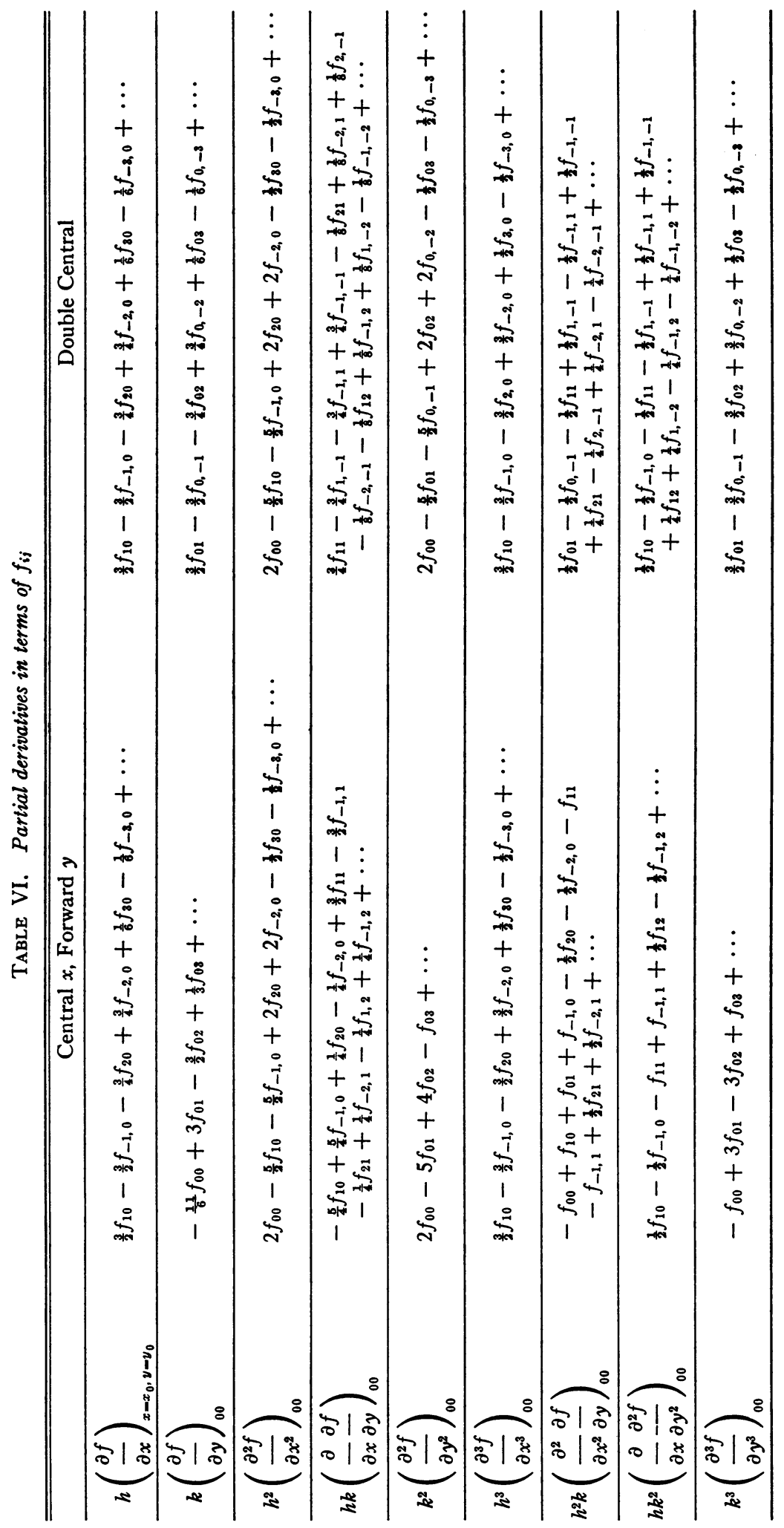


The expressions of the partial derivatives up to the third order have been written out explicitly as shown in Tables V and VI.

Kuo-CHu Ho

University of Florida

Gainesville, Florida

${ }^{1}$ H. E. SAlzer, Jn. Math. and Physics, v. 26, 1948, p. 294-305.

2 H. E. Salzer, Amer. Math. Soc., Bull., v. 51, 1945, p. 279-280.

3 Nautical Almanac, 1937, p. 802.

\section{TECHNICAL NOTES AND SHORT PAPERS}

\section{The First Published Table of Logarithms to the Base Ten}

The first two lines of the title-page of this Table, LOGARITHMORVM/ Chillias Prima., are followed by 26 further lines. The full title is given in JAmes Henderson, Biblioteca Tabularum Mathematicarum, 1926, p. 30; a facsimile of the title-page is given in A. J. Thомpson, Logarithmetica Britannica, part IX, 1924 ; or in his completed work, frontispiece to volume 2, 1954. There is nothing on the title-page to indicate the author, or the date and place of publication of the little 16-page pamphlet, which was, apparently, privately printed. On page 122 of JoHn WARD, The Lives of the Professors of Gresham College, London, 1740, there is the following quotation from a letter, dated 6 December 1617, written by Sir Henry Bourchier to Dr. Usher: "Our kind friend Mr. Briggs hath lately published a supplement to the most excellent table of logarithms, which I presume he sent to you." Thus in connection with other facts the author was revealed to be HENRY BRIGGS (1561-1631) and his table was printed in the latter part of 1617. John Napier (1550-1617) died in the previous April. Briggs had visited Napier in 1615 , spent a month with him in 1616 , and planned to show him this Table in the summer of 1617 .

Copies of the Table are excessively rare; the only copies known to exist are two in the British Museum: (a) with press mark c.54e 10(1); (b) a copy in the Museum's Manuscript Room; and (c) a copy in the Savilian Library, Oxford. (a) and (c) are bound up with Edmund Gunter's Canon Triangulorum, 1623, so that the original may have been trimmed; its present size is $9.3 \times 15.5 \mathrm{~cm}$. A photostat copy of (a) is a recent acquisition of the Library of Brown University.

In this Table are given $\log N, N=[1(1) 1000 ; 14 \mathrm{D}]$, with the first four numbers of first differences, rounded for $N=500$ (1)1000. Characteristics are separated from the decimal parts by lines. The accuracy of this Table is very extraordinary. Every entry of the Table was compared with A. J. Thompson's $\log N$, $N=[1(1) 1000 ; 21 \mathrm{D}]$ and the only errors were 153 in the fourteenth decimal place: 150 unit errors, and 3 two-unit errors at $N=154,239,863$.

In contrast to this, when we turn to Briggs' remarkable Arithmetica Logarithmica, London, 1624, giving $\log N$ for $N=$ [1(1)20000, $90000(1) 10000 ; 14 \mathrm{D}]$ with difference throughout, we find, for $N=1(1) 1000$, no less than 19 errors; 18 two-units in the fourteenth decimal place and one serious error of 6 units in the seventh decimal place. Of the 150 unit errors in the 1617 publication the same 